\title{
Moraxella bovoculi em casos de ceratoconjuntivite infecciosa bovina no Rio Grande do Sul ${ }^{1}$
}

\author{
Felipe Libardoni' ${ }^{2}$, Charles F.C. Scherer ${ }^{3}$, Luana Farias², Andréia Vielmo ${ }^{2}$, Claudia Balzan ${ }^{2}$ \\ e Agueda C. de Vargas ${ }^{2 *}$
}

\begin{abstract}
Libardoni F., Scherer C.F.C., Farias L., Vielmo A., Balzan C. \& Vargas A.C. 2012. [Moraxella bovoculi in cases of infectious bovine keratoconjunctivitis in Rio Grande do Sul, Brazil.] Moraxella bovoculi em casos de ceratoconjuntivite infecciosa bovina no Rio Grande do Sul. Pesquisa Veterinária Brasileira 32(8):743-746. Departamento de Medicina Veterinária Preventiva, Centro de Ciências Rurais, Universidade Federal de Santa Maria, Avenida Roraima 1000, Cidade Universitária, Camobi, Santa Maria, RS 97105-900, Brazil. E-mail: agueda.vargas@gmail.com

Infectious keratoconjunctivitis (IK), although rarely fatal, results in significant economic losses for cattle and sheep farmers. The main causative agents of this disorder are Moraxella bovis and Moraxella ovis. In 2007, a new species also responsible for IK was described. This newly described pathogen, called Moraxella bovoculi, was never reported in Brazil. Therefore, the aim of this study was confirmed the $M$. bovoculi among the samples analyzed. For this, 54 isolates of Moraxella spp. from clinical samples derived from 34 cattle and 18 sheep, sent to the laboratory of bacteriology from 1991 to 2011 was characterized. Differentiation among the species was based on genotypic characteristics, using partial amplification of 16S-23S intergenic region and cleavage products of amplification with enzyme RsaI. Results showed that 25 isolates (46\%) were characterized as M. bovis, 17 (32\%) as M. ovis, and $12(22 \%)$ as M. bovoculi. This means that M. bovoculi is present among cattle herds in Rio Grande do Sul and, therefore, in Brazil.
\end{abstract}

INDEX TERMS: Moraxella bovis, Moraxella ovis, eye disease, polymerase chain reaction, cattle, sheep.

RESUMO.- A ceratoconjuntivite infecciosa (CI), embora raramente fatal, resulta em perdas econômicas significativas para os rebanhos bovinos e ovinos. Os principais agentes causadores dessa enfermidade são Moraxella bovis e Moraxella ovis. Em 2007 foi descrita uma nova espécie também responsável pela $\mathrm{CI}$ e denominada Moraxella bovoculi, que até o presente momento, não havia sido relatada no Brasil. Assim, objetivou-se com este trabalho caracterizar e distinguir 54 isolados de Moraxella spp. de amostras clínicas oriundas de 34 bovinos e 17 ovinos, encaminhadas ao Laboratório de Bacteriologia da Universidade Federal de Santa Maria no período de 1990 a 2011, visando a identificação

\footnotetext{
${ }^{1}$ Recebido em 19 de março de 2012.

Aceito para publicação em 18 de abril de 2012.

${ }^{2}$ Departamento de Medicina Veterinária Preventiva, Centro de Ciências Rurais (CCR), Universidade Federal de Santa Maria (UFSM), Av. Roraima 1000, Cidade Universitária, Bairro Camobi, Santa Maria, RS 97105-900, Brasil. *Autor para correspondência: agueda.vargas@gmail.com

${ }^{3}$ Médico Veterinário, Hipra Saúde Animal Ltda, Avenida do Lami 6133, Porto Alegre, RS 91780-120, Brasil.
}

de $M$. bovoculi. A distinção dos isolados foi fundamentada nas características genotípicas, pela amplificação parcial da região intergênica 16S-23S e clivagem dos produtos da amplificação com enzima RsaI. Como resultados, 25 (46\%) isolados foram caracterizados como M. bovis, 17 (32\%) como M. ovis e 12 (22\%) como M. bovoculi. Logo, conclui-se que $M$. bovoculi encontra-se presente no rebanho bovino do Rio Grande do Sul e, portanto, no Brasil.

TERMOS DE INDEXAÇ̃̃O: Moraxella bovis, Moraxella ovis, doença ocular, ceratoconjuntivite, bovinos, ovinos.

\section{INTRODUÇÃO}

A ceratoconjuntivite infecciosa (CI) é a doença ocular de maior importância para a criação de bovinos e ovinos em todo o mundo (Postma et al. 2008), cuja etiologia primária é atribuída a Moraxella bovis (Henson \& Grumbles 1960) e Moraxella ovis (Spradbrow 1971), respectivamente. 0 gênero Moraxella compreende bactérias Gram-negativas, pleomórficas, aeróbicas e assacarolíticas (Hardie 1986), sendo a diferenciação fenotípica das espécies laboriosa pela alta 
variabilidade das cepas (Fraser \& Gilmour 1979). M. bovis e $M$. ovis são patógenos raramente fatais, que determinam significantes perdas econômicas aos estabelecimentos de criação de bovinos e ovinos (Thiritf \& Overfield 1974, Slatter et al. 1982).

Em 2007 foi descrita uma nova espécie do gênero $M o$ raxella associada à $\mathrm{CI}$ em bovinos, denominada Moraxella bovoculi. A nova espécie foi filogeneticamente relacionada à $M$. bovis e $M$. ovis. Contudo, foi distinguida dos membros do gênero Moraxella pela caracterização bioquímica, sustentada pela análise dos genes constitutivos $16 \mathrm{~S}$, subunidade B da RNA polimerase, hidroxiacil-CoA dehidrogenase, subunidade épsilon da ATP sintase F1, histidina quinase, phospho-N-acetylmuramoyl-pentapeptideo transferase e ferridoxina (Angelos \& Ball 2007, Angelos et al. 2007).

No Brasil, $M$. bovis e $M$. ovis têm sido alvo de relatos e pesquisas (Conceição \& Turnes 2003, Chaves et al. 2008, Carmo et al. 2011), contudo, $M$. bovoculi, até o momento, não foi descrita no país. Logo, objetivou-se com este estudo analisar e identificar genotipicamente os isolados de Moraxella spp. obtidos de amostras clínicas de bovinos e ovinos diagnosticados com CI, enviadas ao Laboratório de Bacteriologia da Universidade Federal de Santa Maria entre os anos de 1990 e 2011, com propósito de identificar M. bovoculi.

\section{MATERIAL E MÉTODOS}

Foram utilizados 54 isolados bacterianos provenientes de 51 amostras da secreção ocular de 34 bovinos e 17 ovinos com $\mathrm{CI}$, de 28 propriedades localizadas em 18 municípios do Rio Grande do Sul (Quadro 1). As amostras foram previamente caracterizadas como Moraxella sp. por meio de análise morfo-tintorial e testes bioquímicos (catalase, oxidase, crescimento em agar MacConkey, produção de indol, motilidade, redução de nitrato, proteólise da caseína e fermentação da glicose) de acordo com Macfaddin (2000), Angelos et al. (2007) e Angelos (2010). Os isolados foram mantidos liofilizados e estocados a $-20^{\circ} \mathrm{C}$ até o momento das análises, quando foram suspendidos e cultivados em meio ágar sangue (sangue ovino 5\%) e incubados em condições de aerobiose a $37^{\circ} \mathrm{C}$ por um período de 48 horas, para realização dos testes morfotintoriais e Fenilalanina Desaminase (FA) para caracterizar a presença de M. bovoculi segundo Angelos et al. (2007). Para comprovação molecular, as células bacterianas foram suspendidas em $1 \mathrm{~mL}$ de água mili-Q para extração de DNA através do protocolo de CTAB (brometo de cetiltrimetil amônio) precedido por uma digestão com $5 \mu \mathrm{L}$ de proteinase $\mathrm{K}(20 \mathrm{mg} / \mathrm{mL})$ por 60 minutos a $37^{\circ} \mathrm{C}$, segundo Sambrook \& Russell (2001).

Para comprovação molecular e diferenciação das espécies de Moraxella, foi realizada a técnica da reação em cadeia da polimerase (PCR) segundo Angelos \& Ball (2007). Foram utilizados os iniciadores ISRup (5'-ACCGACGCTTATCGCAGGTCACTA-3') e ISRdown (5'-GTG TCGAAGCAAAATCAGGGTCGT-3') para amplificação da região intergênica $16 \mathrm{~S}-23 \mathrm{~S}$, com fragmentos de $650 \mathrm{pb}$ para $M$. bovis e $600 \mathrm{pb}$ para $M$. bovoculi e $M$. ovis. A reação da PCR foi realizada em um volume final de $25 \mu \mathrm{L}$, contendo $5 \mu \mathrm{L}$ do tampão, $10 \mu$ mols de cada iniciador, $200 \mu \mathrm{M}$ de cada deoxinucleotídeo trifosfato(dNTP), $1 \mathrm{U}$ de DNA Polimerase GoTaq, $1 \mu \mathrm{L}$ de DNA molde ( 50ng) e água ultrapura. A amplificação foi realizada utilizando-se uma desnaturação inicial por 1 minuto a $95^{\circ} \mathrm{C}$, seguida por 35 ciclos de $30 \mathrm{~s}$ a $95^{\circ} \mathrm{C}, 30 \mathrm{~s}$ a $55^{\circ} \mathrm{C}, 40 \mathrm{~s}$ a $72^{\circ} \mathrm{C}$ e uma extensão final de 4 minutos a $72^{\circ} \mathrm{C}$. Os produtos da amplificação foram verificados em gel de agarose a $1 \%$ corado com brometo de etídio $\left(0,5 \mu \mathrm{g} \mathrm{mL} \mathrm{m}^{-1}\right)$.
Para a distinção das espécies de Moraxella, $10 \mu \mathrm{L}$ do produto da PCR foi submetido à restrição enzimática, com $1 \mathrm{U}$ da enzima RsaI, $18 \mathrm{~mL}$ de água ultra pura e $2 \mathrm{~mL}$ de tampão enzimático, e então incubados a $37^{\circ} \mathrm{C}$ por 2 horas. Após a incubação, $5 \mathrm{~mL}$ da reação de PCR original e $10 \mathrm{~mL}$ da digestão por RsaI foram separados por eletroforese e visualizados em gel de agarose a $2 \%$ corado com brometo de etídio $\left(0,25 \mu \mathrm{g} \mathrm{m}^{-1}\right)$. Segundo Angelos \& Ball (2007) RsaI cliva somente o DNA amplificado de M. bovoculi $(600 \mathrm{pb}) \mathrm{em}$ um único sitio de restrição, resultando em dois fragmentos de aproximadamente 150 e $450 \mathrm{pb}$, e não cliva o fragmento de $M$. bovis $(650 \mathrm{pb})$ nem de $M$. bovoculi $(600 \mathrm{pb})$. Para a confirmação da restrição enzimática, o produto de PCR de algumas amostras foram enviadas para sequenciamento em triplicata na empresa ACT Gene Análises Moleculares Ltda. (Centro de Biotecnologia, UFRGS, Porto Alegre, RS) utilizando o sequenciador automático ABI-PRISM 3100 Genetic Analyzer armado com capilares de $50 \mathrm{~cm}$ e polímero POP6 (Applied Biosystems).

Como controles dos testes fenotípicos e genotípicos, foram utilizadas as cepas padrão ATCC10900 - M. bovis; ATCC19575 - M. ovis e ATCCBAA1259 - M. bovoculi.

\section{RESULTADOS}

Na análise fenotípica morfológica à coloração de Gram, as amostras caracterizadas genotipicamente como Moraxella bovis apresentaram pleomorfismo $(65,38 \%$ cocobacilos, $19,23 \%$ bacilos com formações em pequenas cadeias e $15,38 \%$ diplococos/DC). Todavia $100 \%$ das amostras de $M$. ovis e $M$. bovoculi demonstraram morfologia de diplococos. No teste de atividade da fenilalanina desaminase apenas $12(35,29 \%)$ isolados provenientes de bovinos foram positivos e compatíveis com M. bovoculi (Angelos et al. 2007). Dos 54 isolados submetidos à análise pela PCR da região intergênica 16S-23S, 25 apresentaram amplificação de 650 pb e outros 29 amplificaram de 600 pb. Após a restrição enzimática dos produtos da PCR, 12 isolados tiveram os fragmentos de $600 \mathrm{pb}$ digeridos pela enzima de restrição $R s a$ I resultando em dois fragmentos de aproximadamente 150 e $450 \mathrm{pb}$, confirmando a presença de $M$. bovoculi entre os isolados brasileiros de Moraxella spp. oriundos de bovi-

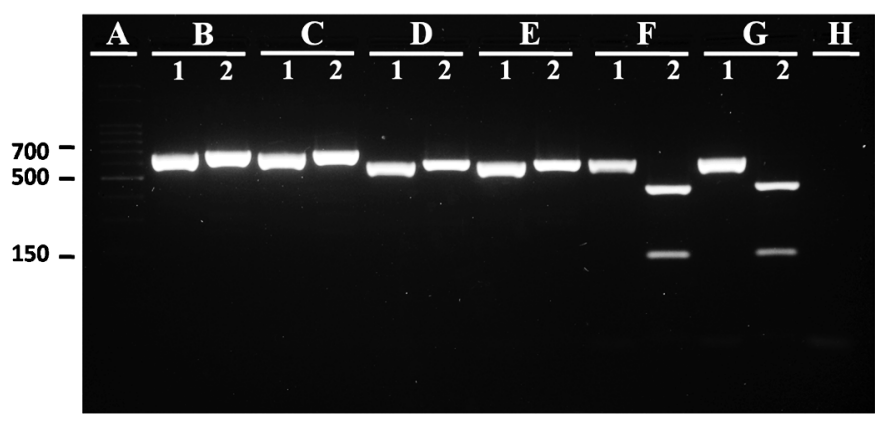

Fig.1. Eletroforese em agarose 2\%. (linha A) marcador de peso molecular de 100pb; (linha B) Moraxella bovis ATCC 10900; (linha C) M. bovis SB 024/90; (linha D) M. ovis ATCC 19575; (linha E) M. ovis SB 270/91; (linha F) M. bovoculi ATCC BAA 1259; (linha G) M. bovoculi SB 82/92 (linha H) controle negativo. (1) Produtos de amplificação da região intergênica 16S-23S. (2) Produto da reação de PCR digerido com RsaI. Os produtos amplificados de $\sim 650 \mathrm{pb}(M$. bovis $)$ e $\sim 600 \mathrm{pb}(M$. ovis $)$ não foram clivados por RsaI. Porém o amplificado de $600 \mathrm{pb}$ de $M$. bovoculi foi clivado uma vez em fragmentos de $\sim 450 \mathrm{pb}$ e $\sim 150$-pb. 
Quadro 1. Distribuição dos municípios estudados e características moleculares das amostras de Moraxella spp. isoladas de ceratoconjuntivite infecciosa bovina e ovina no Rio Grande do Sul

\begin{tabular}{|c|c|c|c|c|c|c|c|}
\hline \multirow[t]{2}{*}{ Registro } & \multirow[t]{2}{*}{ Município } & \multirow[t]{2}{*}{ Ano } & \multirow[t]{2}{*}{ Espécie } & \multirow[t]{2}{*}{$\mathrm{N}^{*}$} & \multicolumn{3}{|c|}{ Caracterização molecular** } \\
\hline & & & & & M. bovis & M. bovoculi & M. ovis \\
\hline SB024/90 & São Martinho da Serra & 1990 & Bovino & 1 & 1 & - & - \\
\hline SB082/92 & Dilermando de Aguiar & 1992 & Bovino & 1 & - & 1 & - \\
\hline SB234/93 & Cacequi & 1993 & Bovino & 1 & 1 & - & - \\
\hline SB030/95 & Tupanciretã & 1995 & Bovino & 1 & - & 1 & - \\
\hline SB156/96 & Lavras do Sul & 1996 & Bovino & 1 & 1 & - & - \\
\hline SB273/96 & Tupanciretã & 1996 & Bovino & 1 & - & 1 & - \\
\hline SB548/99 & São Borja & 1999 & Bovino & 1 & 1 & - & - \\
\hline SB151/01 & Alegrete & 2001 & Bovino & 1 & 1 & - & - \\
\hline SB111/02 & Alegrete & 2002 & Bovino & 1 & 1 & - & - \\
\hline SB $150 / 02$ & Tupaciretã & 2002 & Bovino & 1 & - & 1 & - \\
\hline SB $163 / 03$ & Formigueiro & 2003 & Bovino & 2 & - & 2 & - \\
\hline SB246/04 & Santa Maria & 2004 & Bovino & 1 & 1 & - & - \\
\hline SB021/08 & São Vicente do Sul & 2008 & Bovino & 1 & 1 & - & - \\
\hline SB139/10 & Vitor Graeff & 2010 & Bovino & 1 & - & 1 & - \\
\hline SB150/10 & Dilermando de Aguiar & 2010 & Bovino & 1 & - & - & 1 \\
\hline SBP $15 / 10$ & Pelotas & 2010 & Bovino & 1 & - & 1 & - \\
\hline SBP 04/11 & Restinga Seca & 2011 & Bovino & 7 & 6 & 2 & - \\
\hline SB013/11 & São Gabriel & 2011 & Bovino & 1 & 1 & - & - \\
\hline SB028/11 & Dilermando de Aguiar & 2011 & Bovino & 9 & 9 & 2 & - \\
\hline SB270/91 & São Martinho da Serra & 1991 & Ovino & 2 & - & - & 2 \\
\hline SB249/92 & Cruz Alta & 1992 & Ovino & 3 & - & - & 3 \\
\hline SB567/05 & Santa Maria & 2005 & Ovino & 1 & - & - & 1 \\
\hline SB296/07 & Caçapava do Sul & 2007 & Ovino & 1 & - & - & 1 \\
\hline SB006/08 & Caçapava do Sul & 2008 & Ovino & 1 & - & - & 1 \\
\hline SB007/08 & São Sepé & 2008 & Ovino & 1 & - & - & 1 \\
\hline SB012/10 & Dom Pedrito & 2010 & Ovino & 3 & 1 & - & 2 \\
\hline SB020/11 & São Sepé & 2011 & Ovino & 3 & - & - & 3 \\
\hline SB022/11 & São Martinho da Serra & 2011 & Ovino & 2 & - & - & 2 \\
\hline TOTAL & & & & 51 & $25(46 \%)$ & $12(22 \%)$ & $17(32 \%)$ \\
\hline
\end{tabular}

nos com CI (Quadro 1). Os outros 25 produtos de 650pb e os 17 de 600 pb não foram clivados pela RsaI, caracterizando $M$. bovis e M. ovis, respectivamente (Fig.1).

De acordo com a caracterização genotípica, foram obtidos 25 isolados de M. bovis, 17 de M. ovis e 12 de M. bovoculi. No Quadro 1, visualiza-se a identificação de $M$. bovoculi apenas em amostras oriundas de bovinos em seis dos $18 \mathrm{mu}-$ nicípios dos quais se havia isolado Moraxella spp. de casos de CI. A maioria dos isolados obtidos de bovinos $(70,6 \%)$ foram caracterizados como $M$. bovis e os isolados de ovinos como $M$. ovis (93,3\%), no entanto, uma amostra de $M$. ovis foi isolada de bovino (SB 150/10) e uma amostra de M. bovis foi isolada de ovino (SB 12/10). Visando confirmar os resultados da restrição enzimática, o produto de PCR das amostras SB 150/10 e SB 12/10 foram sequenciados e comparados ao banco de dados Basic Local Alignment Search Tool (BLAST-n), onde apresentaram identidade de $100 \%$ e $99 \%$ respectivamente, com $M$. ovis e M. bovis, confirmando o resultado da restrição enzimática. Além disso, foram isoladas concomitantemente $M$. bovis e $M$. bovoculi em três amostras registradas como SBP04/11 e SB28/11 (Quadro 1).

Quanto às características fenotípicas das colônias isoladas, não foi possível identificar diferenças morfológicas nas amostras que foram reisoladas (anteriores a 2011, estocadas liofilizadas). Porém, nas amostras mais recentes (isoladas em 2011) em que foram identificadas concomitantemente M. bovoculi e M. bovis (Quadro 1), foi possível a visualização de diferenças quanto ao tamanho das colônias, sendo as colônias de $M$. bovoculi de menor diâmetro.

\section{DISCUSSÃO}

O elevado pleomorfismo observado nas amostras de Moraxella spp., nesse estudo, demonstra que a morfologia bacteriana na coloração de Gram é um critério pouco preciso para identificar as espécies cocóides de Moraxella, uma vez que $15,38 \%$ dos isolados de $M$. bovis apresentaram-se como diplococos, concordando com os relatos da presença de bactérias com características de cocos Gram-negativos em surtos de CI descritos há muito tempo na literatura mundial (Wilcox 1970, Fraser \& Gilmour 1979). Além disso, Poels (1917), Fairlie (1966) e Elad et al. (1988) relataram o isolamento de cocos Gram-negativos de bovinos com CI que não causaram doença após inoculação experimental, levantando a suspeita que poderia ser uma terceira espécie de Moraxella envolvida na doença.

Recentemente Angelos et al. (2007) relataram uma nova espécie de Moraxella isolada de bovinos com CI denominada $M$. bovoculi, e indicaram o teste de fenilalanina desaminase (reação positiva) para diferenciá-la de $M$. ovis e $M$. bovis (reações negativas). Esse perfil fenotípico foi corroborado pelos resultados de nosso estudo. Porém Angelos \& Ball (2007) identificaram isolados de $M$. bovoculi fenilalanina desaminase-negativos, e por isso desenvolveram um método molecular para aumentar a eficácia na diferencia- 
ção das espécies de Moraxella. Esse método envolve a reação de PCR para amplificar a região intergênica entre os rRNAs16S e 23S seguido por digestão enzimática do produto amplificado.

A caracterização concordante entre o método molecular e o teste de fenilalanina desaminase, observada no presente estudo, pode ser justificado pela baixa amostragem utilizada e/ou pouca diversidade das amostras. 0 teste de desaminação da fenilalanina é a forma mais econômica de caracterização, porém nos casos em que forem diagnosticados cocos Gram-negativos fenilalanina desaminase negativo em CI, o uso da PCR se faz necessário para obtenção do diagnóstico definitivo (Angelos \& Ball 2007).

Em bovinos foram identificadas $M$. bovis, $M$. bovoculi e $M$. ovis em casos de $\mathrm{CI}$, fato já relatado anteriormente por Elad et al. (1988) e Angelos et al. (2007). No entanto, em ovinos foram identificadas $M$. ovis e $M$. bovis, sendo o achado dessa última espécie, em ovinos, até então não descrita no Brasil. 0 isolamento de $M$. bovis em ovinos e M. ovis em bovinos alerta para a possibilidade de transmissão e prováveis reservatórios desses patógenos quando são utilizados regimes de criação consorciados destas duas espécies nas propriedades.

A identificação de $M$. bovoculi nas amostras isoladas de bovinos nos anos de 1992, 1995 e 1996 demonstra que essa espécie está presente, pelo menos, desde a década de 1990, em casos de ceratoconjuntivite infecciosa bovina em municípios no Rio Grande do Sul, porém não era caracterizada. Mundialmente são escassos os estudos a respeito da ocorrência de M. bovoculi. Na América do Sul, apenas Sossa et al. (2010) relatam surtos de CI causados por M. bovoculi em bovinos.

Apesar de terem sido observadas culturas mistas de $M$. bovis e M. bovoculi em três das 34 amostras analisadas provenientes de bovinos, na maioria dos casos, o isolamento de $M$. bovoculi ocorreu de forma individualizada. Esses resultados vêm ao encontro das evidências clínicas, as quais sugerem que essa espécie recentemente caracterizada desempenha um papel importante na patogênese da CI e, portanto estudos que ressaltem a epidemiologia da doença devem ser realizados (Angelos 2010).

Uma vez que os postulados de Koch não tenham sido estabelecidos para M. bovoculi, acredita-se que nos casos com envolvimento de $M$. bovoculi, sejam necessários fatores adicionais para a ocorrência de doença clínica, assim como ocorre na CI causada por M. bovis. Dentre esses fatores, a raça dos animais, a influência da radiação UV e traumas ou irritações oculares causados por pastagens altas, poeira, vento, e até mesmo estresse de manejo devem ser considerados para que se estabeleça a infecção (Barner 1952,Ward \& Nielson 1979).

Futuros estudos com ampliação da amostragem de casos clínicos de CI poderão elucidar a distribuição geográfica e a variabilidade genética e bioquímica existente entre os isolados de M. bovoculi, bem como avaliar sua ocorrência em outras espécies de animais domésticos que podem servir de reservatórios aos bovinos. Estes estudos poderão subsidiar a modernização das plataformas de produção de vacina na indústria nacional, que não contém $M$. bovoculi em suas formulações.

\section{CONCLUSÃO}

Moraxella bovoculi está presente nos casos clínicos de ceratoconjuntivite infecciosa bovina (CIB) no Rio Grande do Sul, bem como M. bovis e M. ovis. Dessa forma, novas pesquisas que elucidem a participação de M. bovoculi, M. ovis $e$ M. bovis nos casos de ceratoconjuntivite infecciosa em rebanhos são necessárias para a implantação de medidas de controle e prevenção adequadas, visando o benefício da cadeia produtiva da bovinocultura e ovinocultura.

\section{REFERÊNCIAS}

Angelos J.A. \& Ball L.M. 2007. Diferentiation of Moraxella bovoculi sp. nov. from other moraxella by the use of polymerase chain reaction and restriction endonuclease analysis of amplified DNA. J. Vet. Diagn. Invest. 19:532-534

Angelos J.A., Spinks P.Q., Ball L.M. \& George L.W. 2007. Moraxella bovoculi sp. nov. isolated from calves with infectious bovine keratoconjunctivitis. Int. J. Syst. Evol. Microbiol. 7:789-795.

Angelos J.A. 2010. Moraxella bovoculi and infectious bovine keractoconjunctivitis: Cause or coincidence? Vet. Clin. North. Am., Food. Anim. Pract. 26:73-78.

Barner R.D. 1952. A study of Moraxella bovis and its relation to bovine keratitis. Am. J. Vet. Res. 13:132-144.

Carmo P.M.S., Vargas A.C., Rissi D.R., Oliveira-Filho J.C., Pierezan F., Lucena R.B., Leite F.L.L. \& Barros C.S.L. 2011. Surto de ceratoconjuntivite infecciosa bovina e hemoncose causando mortalidade em bezerros. Pesq. Vet. Bras. 31:374-378.

Chaves N.S.T., Lima A.M.V. \& Amaral A.V.C. 2008. Surto de ceratoconjuntivite em ovinos causada por Moraxella spp. no estado de Goiás, Brasil. Ciênc. Anim. Bras. 9:256-261.

Conceição F.R. \& Turnes C.G. 2003. Moraxella bovis: influência das características genotípicas e fenotípicas no controle da Ceratoconjuntivite Infecciosa Bovina. Ciência Rural 33:778-787.

Elad D., Yeruham I. \& Bernstein M. 1988. Moraxella ovis in cases of bovine infectious keratoconjunctivitis (IBK) in Israel. J. Vet. Med. 35:431-434.

Fairlie G. 1966. The isolation of a haemolytic Neisseria from cattle and sheep in the North of Scotland. Vet. Rec. 78:649-650.

Fraser J. \& Gilmour N.J.L. 1979. The identification of Moraxella bovis and Neisseria ovis from the eyes of cattle and sheep. Res. Vet. Sci. 27:127-128.

Hardie J.M. 1986. Bergey's Manual of Systematic Bacteriology. Williams and Wilkins, Baltimore.

Henson J.B. \& Grumbles L.C. 1960. Infectious bovine keratoconjunctivitis. I. Etiology. Am. J. Vet. Res. 51:526-531.

Macfaddin J.F. 2000. Biochemical Testes for Identification of Medical Bacteria. Lippincott, Philadelphia. 912p.

Poels J. 1917. Keratitis infectiosa in cattle (keratitis pyobacillosa). J. Am. Vet. Med. Assoc. 51:526-531.

Postma G.C., Carfagnini J.C. \& Minatel L. 2008. Moraxella bovis pathogenicity: An update. Comp. Immunol. Microbiol. Infect. Dis. 31:449-458.

Sambrook R. \& Russel D.W. 2001. Molecular Cloning: A laboratory manual. $3^{\text {rd }}$ ed. Cold Spring Harbor Laboratory Press, New York.

Sossa V., Cattaneo M., Duran E. \& Zunino P. 2010. Genotypic diversity of Moraxella spp. strains obtained from infectious bovine keratoconjunctivitis cases in Uruguay. Annals Congress of the World Association for Buiatrics, Santiago, Chile.

Spradbrow P. 1971. Experimental infection of the ovine cornea with Neisseria ovis. Vet. Rec. 88:615-616.

Slatter D.H., Edwards M.E., Hawkins C.D. \& Wilcox G.E. 1982. A national survey of the clinical features, treatment and importance of infectious bovine keratoconjunctivitis. Aust. Vet. J. 59:69-72.

Thiritf F.A. \& Overfield F.R. 1974. Impact of pinkeye (infectious bovine kerato-conjunctivitis) on weaning and postweaning performance of Hereford calves. J. Anim. Sci. 38:1179-1184.

Ward J.K. \& Nielson M.K. 1979. Pinkeye (Bovine Infectious Keratoconjunctivitis) in beef cattle. J. Anim. Sci. 49:361-366.

Wilcox G.E. 1970. An examination of Moraxella and related genera commonly isolated from the bovine eye. J. Comp. Pathol. 80:65-74. 